\title{
Skin disorders in preschool environment in the city of Ouagadougou (Burkina Faso): Epidemiological, clinical and therapeutic aspects
}

\author{
Nina Korsaga/Somé1,2, Amina Zoungrana/Ouédraogo, ${ }^{1,3}$, \\ Issouf Konaté4, Muriel Ouédraogo/Ouédraogo ${ }^{1,3}$, Gilbert Patrice Tapsoba ${ }^{1,3}$, \\ Nafissatou Sosso-Kargougou ${ }^{3}$, Fla Koueta', Abou Coulibaly ${ }^{5}$, Jean-Baptiste Andonaba ${ }^{4}$, \\ Pascal Niamba ${ }^{1,3}$, Fatou Barro/Traoré1, Adama Traoré1,3
}

${ }^{1}$ Faculty of Health Science of Joseph Ki-Zerbo University, Ouagadougou, Burkina Faso, ${ }^{2}$ Department of Dermatology and Venereology of Boulmiougou District Hospital, Ouagadougou, Burkina Faso, ${ }^{3}$ Department of Dermatology and Venerology, Yalgado Ouédraogo University Hospital of Ouagadougou, Burkina Faso, ${ }^{4}$ Higher Institute of Health Sciences, Nazi Boni University, Bobo-Dioulasso, Burkina Faso, ${ }^{5}$ Research Institut of health science, Ouagadougou, Burkina Faso

Corresponding author: Dr. Nina Korsaga/Somé, E-mail: nessine2000@yahoo.fr

\begin{abstract}
Background: Preschool children are a vulnerable to skin diseases. However, the epidemiological and clinical profil of these conditions is little studied in this population in Burkina Faso. The purpose of this work was to study the skin conditions for pre-school children in the city of Ouagadougou. Patients et Methods: We conducted a descriptive cross-sectional study from March to June 2017, including children aged 2-6 years, regularly enrolled in a public or private nursery school. We conducted two stages cluster sampling from the list of 23 public awakening and preschool education centers and the 359 deprived of the city of Ouagadougou. The minimum sample size was calculated to 2234 children with Swartzch formula. Epidemiological, clinical and therapeutic variables were sought. Results: Out of 2342 examined children, 792 were presenting a dermatoses, that is a frequency of 33.8 percent. Boys accounted for 50.9 percent. The average age was 4 years. Infectious skin diseases accounted for $33.3 \%$, and the inflammatory $18.4 \%$. The most frequent infectious dermatoses were the folliculitis/boils (18.5\%), ringworm (7.6\%) and dermatophytie of the glabrous skin (2.3\%). Inflammatory and immuno-allergic dermatoses were the sweating Miliaria (17.9\%), Seborrheic Dermatitis (2.1\%) and prurigo (2\%). Genodermatoses and malformations were the naevi (9.6\%), angioma $(0.5 \%)$ and lichen striatus $(0.5 \%)$. Parents were unaware the existence of their children dermatosis in $95.4 \%$ of the cases. Only 36 children had consulted a health officer before the investigation, most often after a traditional treatment failure. No school had an infirmary. Conclusion: dermatological disorders are common in preschool. Also, they are ignored or trivialized by parents and teachers. Awareness campaigns, would allow a screening and early treatment of these conditions.
\end{abstract}

Key words: Skin diseases; Preschool children; Ouagadougou

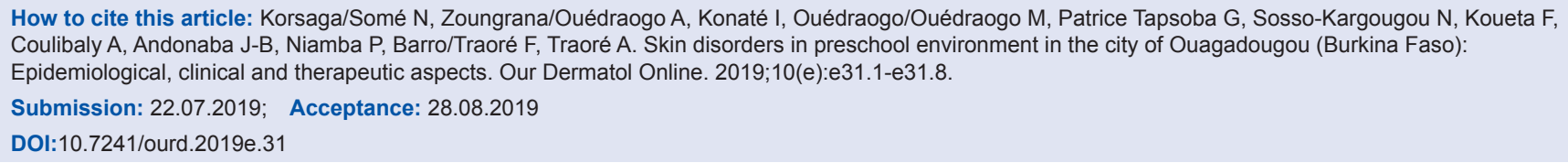




\title{
Dermatoses en milieu préscolaire dans la ville de Ouagadougou (Burkina Faso): Aspects épidémiologique, clinique et thérapeutique
}

\author{
Nina Korsaga/Somé 1,2 , Amina Zoungrana/Ouédraogo, \\ Issouf Konaté4, Muriel Ouédraogo/Ouédraogo ${ }^{1,3}$, Gilbert Patrice Tapsoba ${ }^{1,3}$, \\ Nafissatou Sosso-Kargougou ${ }^{3}$, Fla Koueta', Abou Coulibaly ${ }^{5}$, Jean-Baptiste Andonaba ${ }^{4}$, \\ Pascal Niamba ${ }^{1,3}$, Fatou Barro/Traoré1, Adama Traoré ${ }^{1,3}$
}

${ }^{1}$ Faculty of Health Science of Joseph Ki-Zerbo University, Ouagadougou, Burkina Faso, ${ }^{2}$ Department of Dermatology and Venereology of Boulmiougou District Hospital, Ouagadougou, Burkina Faso, ${ }^{3}$ Department of Dermatology and Venerology, Yalgado Ouédraogo University Hospital of Ouagadougou, Burkina Faso, ${ }^{4}$ Higher Institute of Health Sciences, Nazi Boni University, Bobo-Dioulasso, Burkina Faso, ${ }^{5}$ Research Institut of health science, Ouagadougou, Burkina Faso

Corresponding author: Dr. Nina Korsaga/Somé, E-mail: nessine2000@yahoo.fr

\section{RÉSUMÉ}

Introduction: Les enfants d'âge préscolaire constituent une couche vulnérable aux affections cutanées. Pourtant le profil épidémiologique et clinique de ces affections est peu étudié dans cette population au Burkina Faso. Le but de ce travail était d'étudier les affections cutanées chez les enfants d'âge préscolaire de la ville de Ouagadougou. Patients et Méthodes: Nous avions mené une étude transversale descriptive de mars à juin 2017, incluant les enfants âgés de 2 à 6 ans, régulièrement inscrits dans une école maternelle publique ou privée. Nous avons réalisé un échantillonnage aléatoire en grappe à deux degrés à partir de la liste des 23 Centres d'Eveil et d'Education Préscolaire (CEEP) publics et des 359 CEEP privés de la ville de Ouagadougou. La taille minimale de l'échantillon était calculée à 2234 élèves selon la formule de Swartzch. Les variables épidémiologiques, cliniques et thérapeutiques étaient recherchées. Résultats: Sur 2342 enfants examinés, 792 présentaient une dermatose, soit une fréquence de 33,8\%. Les garçons représentaient 50,9\% des enfants. Lâge moyen était de 4 ans. Les dermatoses infectieuses représentaient $33,3 \%$, et les inflammatoires $18,4 \%$. Les dermatoses infectieuses les plus fréquentes étaient les folliculites/furoncles $(18,5 \%)$, la teigne $(7,6 \%)$ et la dermatophytie de la peau glabre $(2,3 \%)$. Les dermatoses inflammatoires et immunoallergiques étaient la miliaire sudorale (17,9\%), la dermite séborrhéique $(2,1 \%)$ et le prurigo (2\%). Les génodermatoses et malformations étaient les nævi $(9,6 \%)$, les angiomes $(0,5 \%)$ et le lichen striatus $(0,5 \%)$. Les parents d'élèves ignoraient ou banalisaient l'existence de la dermatose de leurs enfants dans 95,4\% des cas. Seuls 36 enfants avaient consulté un agent de santé avant l'enquête, le plus souvent après échec d'un traitement traditionnel. Aucune école ne possédait d'infirmerie. Conclusion: Les affections dermatologiques sont fréquentes chez les enfants d'âge préscolaire. Elles étaient également ignorées et/ou banalisées par les parents et les enseignants. Des campagnes d'information, de sensibilisation et de prévention aussi bien des parents que du personnel enseignant, permettrait le dépistage et la prise en charge précoce des affections dermatologiques chez les enfants d'âge préscolaire à Ouagadougou.

Mots clefs: Dermatoses; Enfants préscolaires; Ouagadougou

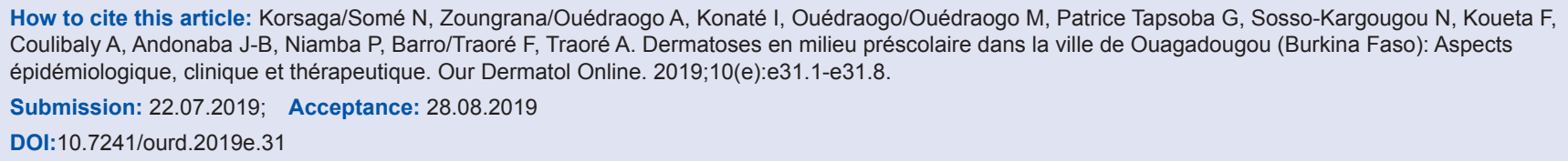




\section{INTRODUCTION}

Au Burkina Faso, les maladies cutanées constituent un problème majeur de santé publique car elles représentent la troisième ou quatrième cause de morbidité dans les formations sanitaires, et constituent 4,7\% des motifs de consultation [1]. Les enfants d'âge préscolaire constituent une couche vulnérable par rapport aux affections cutanées. Les dermatoses pédiatriques sont fréquentes et variées en consultation hospitalière comme le montrent les travaux réalisés en Afrique sur ces dermatoses [2-5].

En effet, les travaux réalisés en Afrique sur les dermatoses pédiatriques sont essentiellement des études faites en milieu hospitalier. Selon ces travaux, 2000 patients consultaient pour une dermatose pédiatrique en 1995 à Lomé au Togo [2] et 1639 cas étaient rapportés à Bamako au Mali en 2012 [6]. Au Burkina Faso, les dermatoses pédiatriques représentaient $21,1 \%$ des consultations au Centre Hospitalier Universitaire (CHU) Yalgado Ouédraogo de Ouagadougou en 1999 [3], et 15,5\% au CHU Sanou Souro de BoboDioulasso entre 2002 et 2008 [4]. Dans le même pays nous disposons de peu de données sur les dermatoses en milieu scolaires. Et ces données qui ne concernent que des affections spécifiques [5,7-9]. Ainsi très peu d'études ont été réalisées en population, sur l'ensemble des dermatoses et chez les enfants d'âge préscolaire.

Ce travail avait donc pour but d'étudier les aspects épidémiologique, clinique et thérapeutique des dermatoses chez les enfants d'âge préscolaire dans la ville de Ouagadougou.

\section{PATIENTS ET MÉTHODES}

\section{Cadre De L'etude}

Notre étude avait pour cadre les établissements préscolaires publics et privés de la ville de Ouagadougou. L e s infrastructures préscolaires dénombrées officiellement étaient 23 Centres d'Eveil et d'Education Préscolaire (CEEP) publics, 359 CEEP privés et 5 Bisongos, répartis dans les 5 communes selon l'ancien découpage administratif de la ville de Ouagadougou [10].

\section{Type Et Periode D'etude}

Nous avons mené une étude descriptive transversale à passage unique, sur la période allant d'avril 2016 à mai 2017. La population d'étude était constituée des enfants des établissements préscolaires de la ville de Ouagadougou.

\section{Echantillonnage et Echantillon}

Nous avons inclus tout élève régulièrement inscrit dans une classe d'un établissement préscolaire public ou privé de la ville de Ouagadougou, présent pendant la période de l'enquête et tiré au sort, sans distinction de sexe et de nationalité. La taille de l'échantillon était obtenue selon la formule de SWARTZCH: $\mathrm{n}_{0}=\mathrm{Z}^{2} \times \mathrm{P} \times \mathrm{Q} / \mathrm{I}^{2}$, avec:

$\mathrm{n}_{0=}$ taille de l'échantillon

$\mathrm{Z}=$ écart réduit

$\mathrm{P}=$ prévalence de référence en $\%$ de la dermatose de l'enfant de 21 mois à 5 ans en milieu hospitalier dans la ville de Ouagadougou (Burkina Faso) en 1999 [3].

$\mathrm{Q}=1-\mathrm{P}$

$\mathrm{I}=$ Marge d'erreur

Pour un seuil de signification de $5 \% \mathrm{Z}=1,96 ; \mathrm{P}=0,12$; $\underline{Q}=0,88 ; \mathrm{I}=0,02$.

$$
\mathrm{n}_{0}=\frac{1,96^{2} \times 0,12 \times 0,88}{0,02^{2}}=1016
$$

Avec un effet de grappe à 2 et un taux de non de réponse de $10 \%$, la taille de l'échantillon était $n=2234$ élèves.

Nous avons ensuite procédé à un échantillonnage en grappe à deux degrés. Au premier degré, nous avons tiré les CEEP (30 au total). Au deuxième degré, nous avons déterminé le nombre d'enfant à enquêter par CEEP par une allocation proportionnelle au nombre d'élèves inscrits dans chaque CEEP (Tab. 1).

\section{Collecte, Analyse Et Ethique}

Les données étaient collectées à l'aide d'une fiche d'enquête comprenant des variables sociodémographiques (âge, sexe), cliniques (durée d'évolution et mode d'installation de la dermatose, signes fonctionnels, signes généraux, topographie des lésions, diagnostics retenus, groupe nosologique, affections non dermatologiques) et thérapeutiques (existence de cadre sanitaire, soins administrés avant l'enquête, traitement proposé pendant l'enquête). La saisie et l'analyse des données étaient effectuées avec le logiciel Epi-Info version 3.1.5, les tableaux, les graphiques et les figures, réalisés avec le logiciel 
Microsoft Word 2010. Lexamen des enfants s'est déroulé dans une salle isolée assurant l'intimité. Seuls les élèves qui acceptaient coopérer étaient examinés. Les pathologies diagnostiquées (dermatologiques ou non) étaient prises en charge par la prescription d'une ordonnance médicale ou la remise d'un bulletin de consultation dans les services intéressés où nous facilitions l'obtention d'un rendez-vous pour le patient.

\section{RÉSULTATS}

Pour ce qui était des aspects épidémiologiques, sur l'échantillon de 2342 enfants examinés, 792 enfants présentaient une dermatose, soit une fréquence $33,8 \%$. Sur ces 792 enfants, nous avons diagnostiqué 880 cas de dermatoses, un enfant pouvant présenter une ou plusieurs dermatoses. Les enfants étaient âgés de 2 à 6 ans avec un âge moyen de 4 ans. Les garçons étaient au nombre de 1199 (50,9\%) et les filles 1143 (48,8\%).

Concernant les aspects cliniques, le mode d'installation des lésions n'était pas connu et la durée d'évolution des dermatoses précisée seulement dans 13 cas, variait de 4 à 60 jours. Le signe fonctionnel le plus fréquent était le prurit dans 24,7\%, suivi de la douleur $(12,7 \%)$, puis du prurit associé à la douleur $(3,2 \%)$. Aucun signe fonctionnel n'était retrouvé dans $59,4 \%$ des cas.

Nous avons regroupé les 880 dermatoses selon 7 groupes nosologiques parmi lesquels, les dermatoses infectieuses étaient les plus fréquentes (33\%), suivies des dermatoses inflammatoires/immuno-allergiques (29\%) (Tab. 2).

Parmi les dermatoses infectieuses, les dermatoses bactériennes venaient en première position avec les folliculites dans 32,\% des cas (Fig. 3), suivies des mycosiques avec la teigne dans $23 \%$ des cas (Fig. 4), des virales et des parasitaires (Tab. 3).

Les dermatoses inflammatoires et immuno-allergiques (Tab. 4) étaient la miliaire sudorale ou sudamina dans $17,93 \%$ des cas (Fig. 5) la dermite séborrhéique $(2,16 \%)$, le prurigo (2,02\%), les eczématides (1,77\%), les eczémas $(1,39 \%)$, la pelade $(0,13 \%)$ et le lichen plan $(0,13 \%)$.

Parmi les génodermatoses et malformations (Tab. 5), les nævi étaient les plus fréquents.

Les pathologies non dermatologiques les plus fréquentes étaient les rhinites $(26,10 \%)$, les bouchons
Tableau 1: Répartition des 2234 enfants enquêtés en fonction de leur effectif par CEEP

\begin{tabular}{|c|c|c|c|c|}
\hline $\mathbf{N}^{\circ}$ & CEEP & Effectif & Proportion & Echantillon \\
\hline 1 & CEEP Secteur 29 & 247 & 0,06 & 136 \\
\hline 2 & CEEP Veenem Nooma & 138 & 0,03 & 76 \\
\hline 3 & $\begin{array}{l}\text { CEEP Namalgbzanga } \\
\text { secteur } 22\end{array}$ & 206 & 0,05 & 114 \\
\hline 4 & $\begin{array}{l}\text { CEEP Privé Piindi (les } \\
\text { Fleurs) }\end{array}$ & 36 & 0,01 & 20 \\
\hline 5 & $\begin{array}{l}\text { CEEP "Le petit Poucet" de } \\
\text { Goughin }\end{array}$ & 66 & 0,02 & 36 \\
\hline 6 & $\begin{array}{l}\text { CEEP Privé la Voie du } \\
\text { succès }\end{array}$ & 121 & 0,03 & 67 \\
\hline 7 & CEEP Scherikidz Cottage & 56 & 0,01 & 31 \\
\hline 8 & CEEP la Plénitude & 118 & 0,03 & 65 \\
\hline 9 & CEEP 1200 Logements & 109 & 0,03 & 60 \\
\hline 10 & CEEP secteur 21 & 290 & 0,07 & 160 \\
\hline 11 & CEEP Cité an II & 170 & 0,04 & 94 \\
\hline 12 & CEEP Municipal & 103 & 0,02 & 57 \\
\hline 13 & CEEP secteur 20 & 175 & 0,04 & 97 \\
\hline 14 & CEEP secteur30 & 182 & 0,04 & 100 \\
\hline 15 & CEEP Pougain Guesba & 102 & 0,02 & 56 \\
\hline 16 & CEEP Mon Eveil & 245 & 0,06 & 135 \\
\hline 17 & CEEP Secteur 9 & 243 & 0,06 & 134 \\
\hline 18 & CEEP Mimpaasgo & 226 & 0,06 & 125 \\
\hline 19 & CEEP Secteur 23 & 177 & 0,04 & 98 \\
\hline 20 & CEEP Niokoll & 121 & 0,03 & 67 \\
\hline 21 & CEEP de Poulesgo & 115 & 0,03 & 63 \\
\hline 22 & CEEP Nonglem Vuillermoz & 75 & 0,02 & 41 \\
\hline 23 & CEEP Sagesse Africaine & 65 & 0,01 & 36 \\
\hline 24 & CEEP la SCOB & 95 & 0,02 & 52 \\
\hline 25 & Kandara & 152 & 0,04 & 84 \\
\hline 26 & Musique sans frontière & 97 & 0,02 & 54 \\
\hline 27 & Prairie & 121 & 0,03 & 67 \\
\hline 28 & WendPanga & 68 & 0,02 & 38 \\
\hline 29 & $\begin{array}{l}\text { CEEP School Wend la } \\
\text { bangré }\end{array}$ & 30 & 0,01 & 17 \\
\hline 30 & Le royaume des tous petits & 100 & 0,02 & 55 \\
\hline Total & & 4049 & 1 & 2234 \\
\hline
\end{tabular}

Tableau 2: Répartition des 880 dermatoses selon le groupe nosologique

\begin{tabular}{lcc}
\hline Groupe nosologique & Effectif (n) & Pourcentage \\
\hline Dermatoses infectieuses & 291 & 33,0 \\
Inflammatoires/ & 255 & 29,0 \\
immuno-allergiques & 127 & 14,5 \\
Lésions cicatricielles [Fig. 1] & 98 & 11,1 \\
Génodermatoses et malformations & 59 & 6,7 \\
Lésions traumatiques & 42 & 4,8 \\
$\begin{array}{l}\text { Dermites parakératosiques des } \\
\text { pieds [Fig. 2] }\end{array}$ & 8 & 0,9 \\
Lésions muqueuses (langue & & $\mathbf{1 0 0 , 0}$ \\
fauchée/géographique) & $\mathbf{8 8 0}$ & \\
Total & &
\end{tabular}

de cérumens $(12,91 \%)$, les conjonctivites $(10,99 \%)$, amygdalite $(10,71 \%)$, et les caries dentaires $(9,89 \%)$

Sur le plan thérapeutique, aucun des 30 CEEP visités ne possédait une infirmerie ni de service d'hygiène scolaire. Certaines écoles disposaient de quelques 


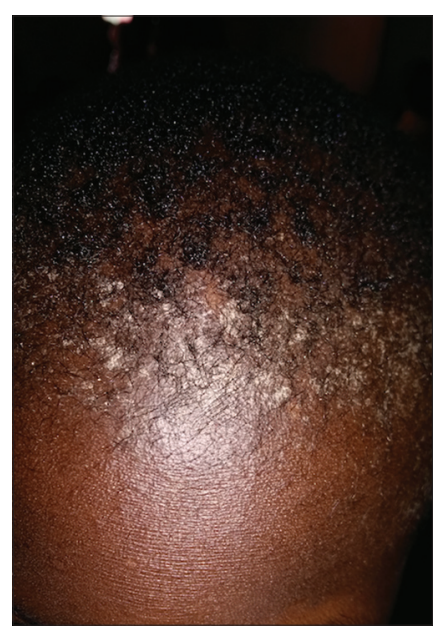

Figura 1: Cicatrice maculeuse dyschromique de l'abdomen suite à une brûlure par eau chaude.

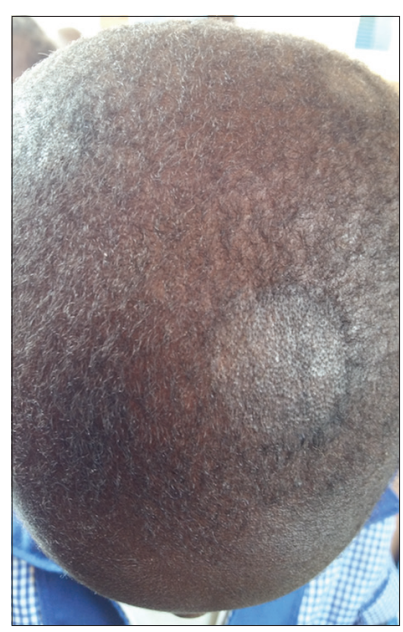

Figura 2: Dermite parakératosique sur fond de lichénification du dos des pieds (ichtyose ?).

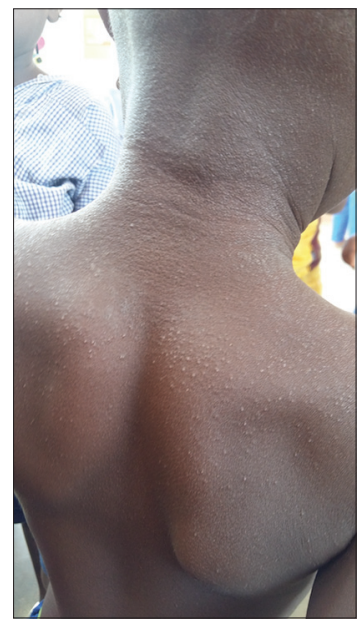

Figura 3: Folliculites de la lisère frontale du cuir chevelu sur fond de miliaire sudorale.

médicaments de base tels que les antalgiques/ antipyrétiques, les anti-diarrhéiques, les antibiotiques

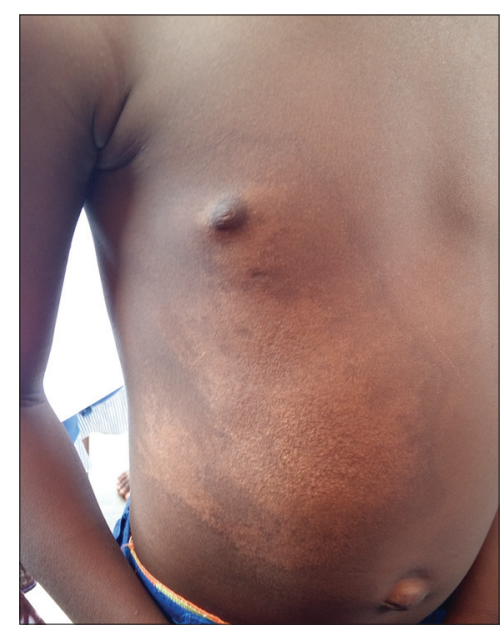

Figura 4: Teigne à grandes plaques alopéciques (microsporique).

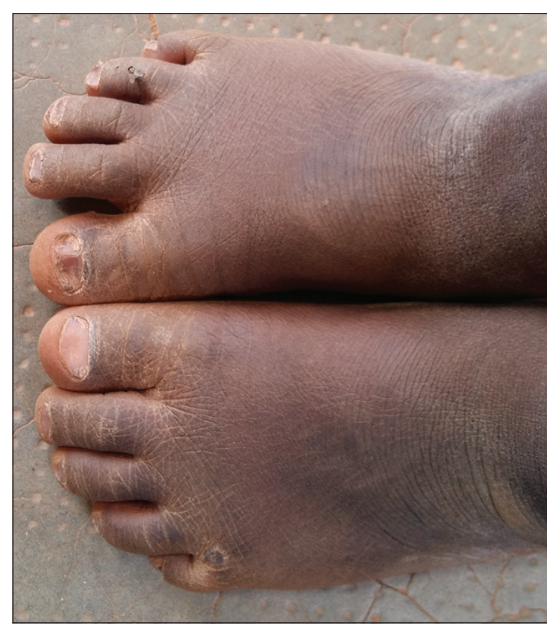

Figura 5: Lésions de miliaire sudorale du dos et de la face postérieure du cou.

(amoxicilline, cotrimoxazole), les antiseptiques locaux (polyvidone iodée) et du coton hydrophile. Sur le plan de l'itinéraire thérapeutique avant l'enquête, seuls 36 enfants $(4,5 \%)$ avaient reçu un traitement, dont 2 après une consultation chez un dermatologue. La plupart des affections étaient d'abord traitées traditionnellement avec des décoctions à boire ou de la poudre (contenant un principe actif non connu des parents) mélangée à du beurre de karité pour application locale. Dans 89\% des cas de dermatoses dépistées, nous avons proposé un traitement dont des antiseptiques locaux dans 19,44\%. Labstention thérapeutique était de 25,89\% et concernait les pathologies déjà sous un traitement adapté ou les affections congénitales.

\section{DISCUSSION}

Les difficultés de précision du début exacte des affections par les parents et la rareté des données 
Tableau 3: Répartition des 291 dermatoses infectieuses selon la fréquence

\begin{tabular}{|c|c|c|c|c|c|}
\hline Groupes nosologiques & Dermatoses & Affections & Effectif & $\begin{array}{l}\% \text { parmi les } 291 \text { dermatoses } \\
\text { infectieuses }\end{array}$ & $\begin{array}{c}\% \text { parmi les } 880 \\
\text { dermatoses }\end{array}$ \\
\hline \multirow[t]{17}{*}{ Dermatoses Infectieuses } & Bactériennes & Folliculite & 91 & 32,3 & 10,3 \\
\hline & & Furoncle & 56 & 19,2 & 6,4 \\
\hline & & Panaris & 06 & 2,0 & 0,7 \\
\hline & & Impétigo & 04 & 1,4 & 0,4 \\
\hline & & Total bactériennes ${ }^{1}$ & 157 & 53,9 & 17,8 \\
\hline & Mycosiques & Teigne & 67 & 23,0 & 7,6 \\
\hline & & Dermatophytie peau glabre & 20 & 6,9 & 2,3 \\
\hline & & Muguet & 07 & 2,4 & 0,8 \\
\hline & & Pityriasis versicolor & 04 & 1,4 & 0,4 \\
\hline & & Total mycosiques ${ }^{2}$ & 98 & 33,7 & 11,1 \\
\hline & Virales & Varicelle & 14 & 4,8 & 1,6 \\
\hline & & Verrues planes & 13 & 4,5 & 1,5 \\
\hline & & Herpès labial & 03 & 1,0 & 0,3 \\
\hline & & PRG & 02 & 0,7 & 0,2 \\
\hline & & Total dermatoses virales ${ }^{3}$ & 32 & 11,0 & 3,6 \\
\hline & Parasitaires & Leishmaniose & 04 & 1,4 & 0,4 \\
\hline & & Total parasitaires ${ }^{4}$ & 04 & 1,4 & 0,4 \\
\hline \multicolumn{3}{|c|}{ Total dermatoses infectieuses ${ }^{1+2+3+4}$} & 291 & 100,0 & 33,0 \\
\hline
\end{tabular}

Tableau 4: Répartition des 255 Dermatoses inflammatoires et immuno-allergiques selon la fréquence

\begin{tabular}{|c|c|c|c|c|c|}
\hline Groupes nosologiques & Dermatoses & Affections & Effectif & $\begin{array}{c}\% \text { parmi les } 255 \\
\text { dermatoses }\end{array}$ & $\begin{array}{c}\% \text { parmi les } 880 \\
\text { dermatoses }\end{array}$ \\
\hline \multirow[t]{14}{*}{ Dermatoses inflammatoires et immuno-allergiques } & Inflammatoires & Sudamina & 158 & 62,0 & 18,0 \\
\hline & & Dermite séborrhéique & 19 & 7,4 & 2,2 \\
\hline & & Prurigo & 18 & 7,0 & 2,0 \\
\hline & & Intertrigo & 17 & 6,7 & 1,9 \\
\hline & & Eczématides & 16 & 6,3 & 1,8 \\
\hline & & Chéilite fissuraire & 10 & 3,9 & 1,1 \\
\hline & & Onyxis & 03 & 1,2 & 0,3 \\
\hline & & Total inflammatoires ${ }^{1}$ & 241 & 94,5 & 27,3 \\
\hline & Allergiques & Eczéma de contact & 10 & 3,9 & 1,1 \\
\hline & & Dermatite atopique & 02 & 0,8 & 0,2 \\
\hline & & Total allergiques ${ }^{2}$ & 12 & 4,7 & 1,3 \\
\hline & Auto-immunes & Pelade & 01 & 0,4 & 0,1 \\
\hline & & Lichen plan & 01 & 0,4 & 0,1 \\
\hline & & Total auto-immunes ${ }^{3}$ & 02 & 0,8 & 0,2 \\
\hline \multicolumn{3}{|c|}{ Total dermatoses inflammatoires et immune-allergiques ${ }^{1+2+3}$} & 255 & 100,0 & 29,0 \\
\hline
\end{tabular}

Tableau 5 : Répartition des 98 Génodermatoses et malformations selon la fréquence

\begin{tabular}{|c|c|c|c|c|c|}
\hline Groupes nosologiques & Dermatoses & Affections & Effectif & $\begin{array}{c}\% \text { parmi les } 98 \\
\text { G et } \mathbf{M}\end{array}$ & $\begin{array}{c}\% \text { parmi les } 880 \\
\text { dermatoses }\end{array}$ \\
\hline \multirow{8}{*}{$\begin{array}{l}\text { Génodermatoses et malformations } \\
\text { (G et } M)\end{array}$} & Génoderma-toses & Nævi & 85 & 86,7 & 9,7 \\
\hline & & Lichen striatus & 04 & 4,1 & 0,4 \\
\hline & & Albinisme & 02 & 2,0 & 0,2 \\
\hline & & Total géndermatoses ${ }^{1}$ & 91 & 92,8 & 10,3 \\
\hline & & Angiome & 04 & 4,1 & 0,45 \\
\hline & Malforma-tions & $\begin{array}{l}\text { Fente labio-palatine } \\
\text { réparée }\end{array}$ & 02 & 2,0 & 0,22 \\
\hline & & $\begin{array}{l}\text { Mamelons } \\
\text { surnuméraires }\end{array}$ & 01 & 1,1 & 0,11 \\
\hline & & Total malformations ${ }^{2}$ & 07 & 7,2 & 0,8 \\
\hline \multicolumn{3}{|c|}{ Total génodermatoses et malformations $\mathbf{s}^{1+2}$} & 98 & 100,0 & 11,1 \\
\hline
\end{tabular}

sur notre sujet dans la littérature, constituaient les principales limites de notre étude. Néanmoins, elle

(c) Our Dermatol Online e.2019 a permis d'apprécier les principales caractéristiques épidémiologique, clinique et thérapeutique des 
dermatoses de l'enfant en milieu préscolaire à Ouagadougou au Burkina Faso.

Ce travail retrouvait une prévalence élevée des dermatoses en milieu préscolaire, sans prédominance de sexe, avec une prépondérance des dermatoses infectieuses et inflammatoires dont les miliaires sudorales, les folliculites/furoncles, les teignes, et très peu d'eczéma et de gale/leishmaniose. Nous avons également noté un nombre important de lésions cicatricielles post-traumatiques. Le prurit était très présent.

Les aspects particuliers étaient le manque de demande soin malgré la présence du prurit, la méconnaissance de l'existence de la dermatose, la négligence des parents et du personnel d'encadrement ainsi que le manque d'infirmerie dans les CEEP.

La fréquence des dermatoses était similaire à celle de Mahé et al., qui au Mali retrouvait une fréquence de 32,2\% en 1997 [11]. Ait Ichen à Taza au Maroc [12] retrouvait un taux supérieur avec 43,16\% de dermatoses en milieu scolaire en 1992. Ces données concordaient avec les données générales de la littérature, qui varient de 21 à $87 \%$ selon les zones géographiques [13].

La prédominance de dermatoses infectieuses était également constatée par plusieurs autres auteurs. En effet Traoré [3] et Kaboré [14] au Burkina, retrouvaient aussi une prédominance de l'atteinte bactérienne respectivement en $1999(39,50 \%)$ et 2014 (46,8\%). Tounkara en Guinée [6] retrouvait une prépondérance des atteintes mycosiques avec 45,74 \% de cas. Bisht, en Inde, trouvait une prédominance des atteintes virales [15]. L'atteinte bactérienne prédominante était à type de pyodermite. Limmaturité du système immunitaire, les conditions socioéconomiques défavorables et environnementales pourraient être les causes de survenue des dermatoses bactériennes dans ce groupe d'âge. La fréquence des infections cutanées chez ces enfants s'explique par le risque élevé de contamination interhumaine de certaines de ces dermatoses avec une propagation rapide dans les collectivités d'enfant pouvant parfois prendre l'aspect d'épidémie $[1,16$,$] . La prévalence des dermatoses$ mycosiques se rapprochait de celle des travaux d'autres auteurs $[6,11,17]$ et montrait l'importance des dermatoses mycosiques dans le milieu préscolaire. Ogunbiyi au Nigéria retrouvait aussi une prédominance des dermatophyties et du pityriasis versicolor en 2005 [18]. La promiscuité en serait-elle la cause?
La fréquence des dermatoses virales se rapprochait des $10,7 \%$ et $7,1 \%$ observés par Traoré au Burkina Faso [3] et Tchangaï-Walla au Togo [2]. La fréquence des infections virales chez l'enfant s'explique par leur contamination interhumaine et l'immaturité immunitaire. Si chez l'adulte, certaines viroses cutanées ont une valeur prédictive positive pour la séropositivité VIH, chez les enfants elles sont souvent banales. Nous n'avons noté aucun cas de gale ni de pédiculose, contrairement aux résultats de Traoré qui, dans le même pays, 18 ans auparavant classaient les parasitoses cutanées à la seconde place des dermatoses infectieuses avec $32,9 \%$, la gale venant en tête de liste. Y'aurait-il une amélioration des conditions climatique, écologique, d'hygiène et socio-économique justifiant ce recul significatif ? La rareté de la leishmaniose serait-elle liée au bas âge des enfants ? En effet, malgré que le Burkina Faso soit une zone d'endémie leishmanienne [1], les études hospitalières retrouvaient très peu d'enfants atteints de leishmaniose $(8,29 \%$ de 0 à 9 ans) [19].

La fréquence élevée des lésions cicatricielles et surtout de cicatrices de brûlure s'expliquait par les d'accidents domestiques. C'est le lieu de rappeler les règles de précaution (toujours mélanger l'eau froide à l'eau chaude, tenir hors de la portée des enfants les repas chauds).

Contrairement à nos résultats, plusieurs autres auteurs Brésiliens et Africains retrouvaient une fréquence plus élevée de dermatoses inflammatoires et immunoallergiques [14,16,20], la différence pourrait s'expliquer par la tranche d'âge de notre étude limitée à 2-6 ans. Le nombre peu élevé de cas d'eczéma, classiquement plus fréquents dans cette tranche d'âge pourrait s'expliquer par leur mode évolutif fait de poussée/ rémission (beaucoup d'enfants étaient-ils en rémission à notre passage ?).

Nous n'avons pas trouvé d'explication à la présence de cette desquamation asymptomatique des pieds chez plusieurs enfants (lésions post-inflammatoire, marche pied nus, climat?).

Lignorance et/ou banalisation de ces dermatoses par les parents d'élèves pourrait être liée au caractère «bénin» des dermatoses dans notre contexte, de plus le recours aux structures de santé ne se fait que très tardivement devant les formes évoluées et après un passage préalable chez le tradipraticien, les croyances et traditions tenant une place importante dans certaines 
familles. L'inaccessibilité financière et géographique pourrait également être un frein au recours aux structures de santé. Labsence d'une infirmerie et/ou d'un service d'hygiène scolaire dans tous les 30 CEEP nous interpelle sur la négligence de la santé des enfants en bas âge par les éducateurs.

\section{CONCLUSION}

Malgré la fréquence élevée des affections dermatologiques chez les enfants d'âge préscolaire, les parents d'élèves ignoraient ou banalisaient ces dermatoses et très peu de parents consultaient un agent de santé. Aucune école ne possédait d'infirmerie. La mise en place d'une stratégie avancée allant vers cette population permettrait le dépistage et la prise en charge de ces affections. Des campagnes d'information, de sensibilisation et de prévention aussi bien des parents que du personnel enseignant permettraient le dépistage et la prise en charge précoce des affections dermatologiques chez les enfants d'âge préscolaire à Ouagadougou.

\section{Statement of Human and Animal Rights}

All procedures followed were in accordance with the ethical standards of the responsible committee on human experimentation (institutional and national) and with the Helsinki Declaration of 1975, as revised in 2008.

\section{Statement of Informed Consent}

Informed consent was obtained from all patients for being included in the study.

\section{RÉFÉRENCES}

1. Direction générale de l'information et des statistiques sanitaires, Annuaire statistique sanitaire 2014 [en ligne]. 2015 mai [consulté le 11/05/2017]: [317 p]. Consultable à l'URL: http://www.insd.com

2. Tchangaï-Walla K, Pitché P, Agbéré A, Bakondé B. Les Motifs de consultations des enfants en dermatologie à Lomé (Togo). Med Afr Noire 1995; 42:391-2.

3. Traoré A, Kouéta F, Sanou I, Kam L, Dao L, Barro F, et al. Les dermatoses courantes de l'enfant dans un service de dermatologie en milieu tropical. CHU de Rouen [En ligne] 1999 Décembre [consulté le 14 juin 2015]: [7 p]. Consultable à l'URL: http://www. chu-rouen.fr/chnpo/Annales/Pubped9.htm

4. Andonaba JB, Diallo B, Sakana L, Niamba P et coll. Aspects épidémiologiques des affections dermatologiques au Centre
Hospitalier Universitaire Souro Sanou de Bobo-Dioulasso. Ann Afr Med. 2010;4:668-77.

5. Compaoré L. Etude des teignes du cuir chevelu en milieu scolaire dans la ville de Ouagadougou (Burkina Faso) [Thèse: Med]. Ouagadougou: Université de Ouagadougou UFR/SDS; 1993: 110p.

6. Tounkara TM, Soumah MM, Keita M, Diané B. Profil épidémiologique et clinique des dermatoses infectieuses chez les enfants au service de dermatologie de l'hôpital national Donka. Ann Dermatol Venereol. 2012;139:137-8.

7. Ouédraogo SM. Etude de la gale humaine en milieu scolaire de la ville de Ouagadougou (Burkina Faso) [Thèse: Med]. Ouagadougou: Université de Ouagadougou Faculté des Sciences de la Santé (FSS); 1997:101p.

8. Traoré A, Korsaga-Somé N, Niamba P, Barro F, Sanou I, Drabo YJ. Pityriasis Rosé de Gibert dans les écoles secondaires à Ouagadougou (Burkina Faso). Ann Dermatol Venereol. 2001;128:605-9.

9. Ouédraogo MS. Aspects épidémiologiques et cliniques de l'acné en milieu scolaire de la ville de Ouagadougou (Burkina Faso) [Thèse: Med]. Ouagadougou: Université de Ouagadougou UFR/SDS; 2001: 101p.

10. Annuaire statistique et répertoire des structures préscolaires 2014/2015. 7eme édition. Unicef, Burkina Faso [en ligne] 2014 Novembre [consulté le 20/07/2015]: [242 p]. Consultable à l'URL: http://www.insd.com

11. Mahé A, Faye O. Projet pilote de lutte contre les maladies de la peau au Mali. Int J Dermatol 1997;36:163-4.

12. Zakaria AI. Enquête épidémiologique des principales dermatoses en milieu scolaire à Taza [Thèse: Med]. Rabat: Université Mohamed VI, Faculté de Médecine et de Pharmacie; 1992: 102p.

13. World Health Organization (WHO). Epidemiology and management of common skin diseases in children in developing countries. [en ligne] 2005 [consulté le 18 octobre 2015]. Consultable à l'URL: http://www.who.int

14. Kaboré HTF. Aspects épidémiologiques et cliniques de la pathologie dermatologique de l'enfant dans le service de DermatologieVénérologie du CHU-YO de 2007 à 2011. [Thèse: Pharm]. Ouagadougou: Université de Ouagadougou, UFR/SDS; 2014: 99p.

15. Bisht JS, Rana SK, Kumari N, Aggarwal B, Mehta A, Singh R. Pattern of dermatoses in preschool children in a teaching hospital in Uttarakhand, India. Indian J Paediatr Dermatol. 2015;16:198-202.

16. Adegbidi $\mathrm{H}$, Padonou F. Profil des dermatoses immunoallergiques chez les enfants dans le service de dermatologie du CNHU-C (Bénin). Méd Santé Trop. 2014;24:446-8.

17. Lightburn E, Morand JJ, Garnotel E, Kraemer P, Meynard JB, Hovette $\mathrm{P}$, et al. Panorama clinique des leishmanioses tégumentaires du Nouveau Monde. Med Trop. 2002;62:637-56.

18. Ogunbiyi AO, Owoaje E, Ndahi A. Prevalence of skin disorders in School Children in Ibadan (Nigeria). Pediatr Dermatol. 2005;22:6-10.

19. Broek I, Harris N, Hekens M, Mekaoui H, Palma P, Szumili E et al. Guide clinique et thérapeutique, Médecins Sans Frontière, Edition Tsunami, Paris: 2010.

20. Ferreira FR, Nascimento LF, Cirvidiu DC. Prevalence of pediatric dermatoses in a university hospital in southeastern Brazil. An Bras Dermatol. 2011;86:477-82.

Copyright by Nina Korsaga/Somé, et al. This is an open-access article distributed under the terms of the Creative Commons Attribution License, which permits unrestricted use, distribution, and reproduction in any medium, provided the original author and source are credited. Source of Support: Nil, Conflict of Interest: None declared. 